\title{
OAERP: a better measure than accuracy in discriminating a better solution for stochastic classification training
}

\begin{abstract}
The use of accuracy metric for stochastic classification training could lead the solution selecting towards the sub-optimal solution due to its less distinctive value and also unable to perform optimally when confronted with imbalanced class problem. In this study, a new evaluation metric that combines accuracy metric with the extended precision and recall metrics to negate these detrimental effects was proposed. This new evaluation metric is known as Optimized Accuracy with Extended Recall-precision (OAERP). By using two examples, the results has shown that the OAERP metric has produced more distinctive and discriminating values as compared to accuracy metric. This paper also empirically demonstrates that Monte Carlo Sampling (MCS) algorithm that is trained by OAERP metric was able to obtain better predictive results than the one trained by the accuracy metric alone, using nine medical data sets. In addition, the OAERP metric also performed effectively when dealing with imbalanced class problems. Moreover, the t-test analysis also shows a clear advantage of the MCS model trained by the OAERP metric against its previous metric over five out of nine medical data sets. From the abovementioned results, it is clearly indicates that the OAERP metric is more likely to choose a better solution during classification training and lead towards a better trained classification model.
\end{abstract}

Keyword: Accuracy; Evaluation metric; Hybrid evaluation metric; Precision; Recall; Stochastic classification model 